resulting effluent is divided into two. In one stream, the peptides are mixed with the colour-developing reagent, while in the other the peptides are hydrolysed into their constituent amino acids before colour development. By the insertion of a timedelay coil in the unhydrolysed stream, the two effluents arrive at their respective colorimeters at the same time and are recorded simultaneously on a 2 -pen recorder. The chromogen generally reacts with peptides only at their terminal amino group, whereas each of the liberated amino acids reacts. Therefore a comparison of the peaks given by the unhydrolysed and hydrolysed streams yields a measure of the number and size of the peptides present in the sample. In spite of the difficulties encountered with this system it has possible applications in the analysis of biological materials.

\title{
REFERENCE
}

Catravas, G. N. (1964). Analyt. Chem, 36, 1146.

\section{Determination of total amino acid concentrations in plasma}

\section{By J. S. Slater and E. Joan Dunnett, Moredun Research Institute, Gilmerton, Edinburgh}

An automated method has been developed for the direct measurement of plasma total amino acid concentrations using a ninhydrin-hydrazine reagent (Research Bulletin no. 20, Technicon Instruments Ltd). The analytical system is shown on Fig. I.

Plasma $(0.1 \mathrm{ml})$ is diluted $\mathrm{I}: \mathrm{I} 0$ with $0.85 \% \mathrm{NaCl}$ containing $0.5 \% \mathrm{BRIJ}_{35}$ and dialysed against the same saline solution. Standards are introduced hourly and alternate cups filled with saline to improve wash out. The rate of analysis is sixteen samples/h. Replicate analysis on the same plasma at varying dilutions gave a total amino acid concentration of $3.04 \pm 0.15 \mathrm{mM}$ as norleucine (20). Interference by urea is negligible and ammonia contributes less than $2 \%$ of the total colour at the concentrations found in fasting plasma.

Diffusion of individual amino acids through the dialyser membrane varied between $33 \%$ and $4 \mathrm{I} \%$. Colour yields varied considerably but all identifiable plasma amino acids showed a linear relationship between concentration and absorbance up to optical densities of $\mathrm{r} \cdot \mathrm{O}$.

This method has been used extensively in studies of ovine maternal and foetal plasma. Results may be corrected to give an approximation of the true total amino acid concentration using the weighted mean norleucine equivalent (Prenton \& London, 1967). In comparison with values obtained by ion exchange chromatography, recoveries of total amino acids by the present method were: maternal plasma I06.I $\pm 4 \cdot 0 \%$ (I 2 ), foetal plasma I02. I $\pm 7 \cdot 2 \%$ ( 13 ).

In maternal and foetal samples taken simultaneously from anaesthetized ewes 


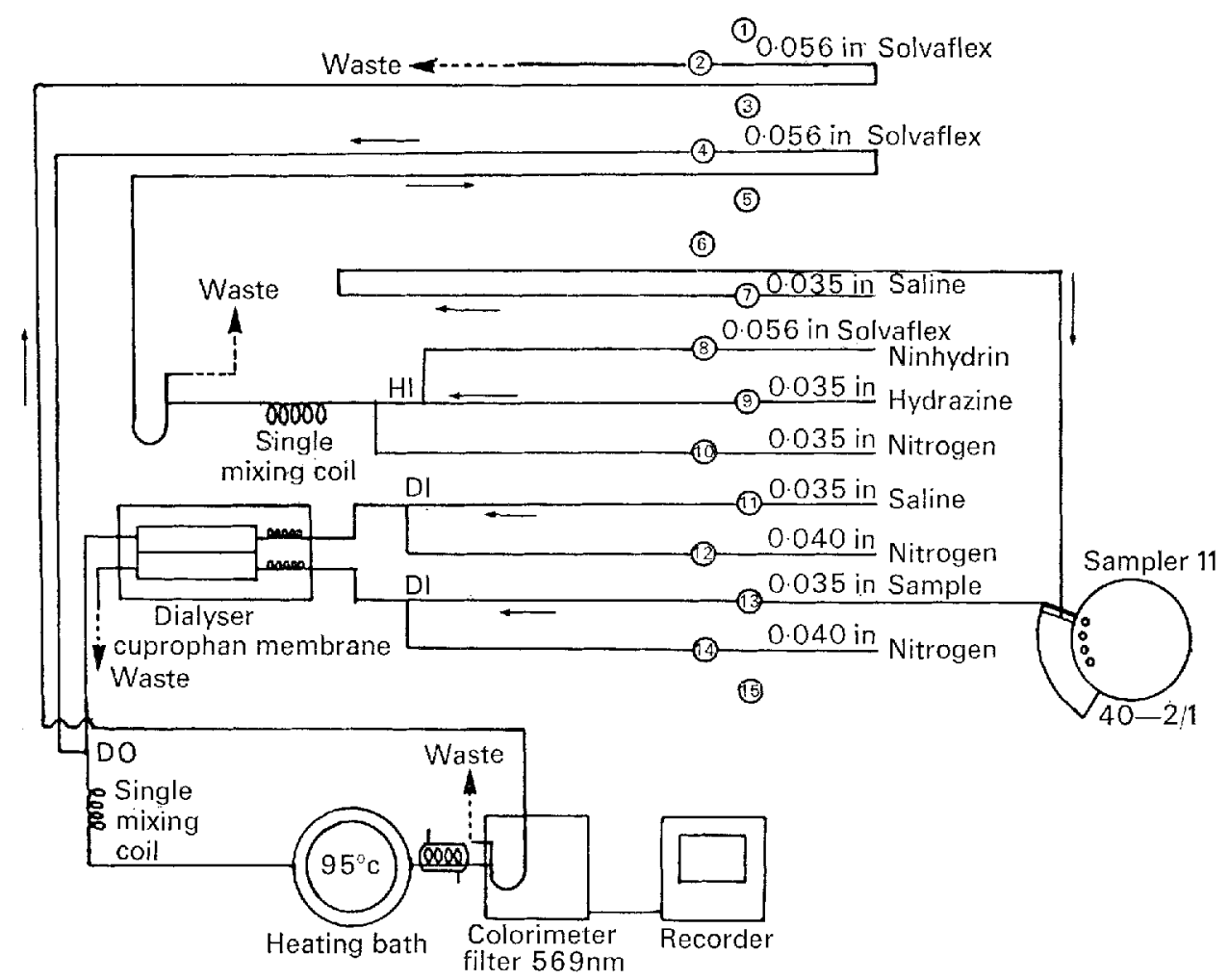

Fig. I. Hlow diagram for determination of total amino acid concentrations.

in the latter half of gestation the average total amino acid concentrations found were: maternal arterial plasma $3.34 \pm 0.63$ (19), umbilical venous plasma $6 \cdot 28 \pm 2 \cdot 32$ (30), umbilical arterial plasma $7 \cdot 25 \pm 2 \cdot 13(30)$ (Hervey \& Slater, in preparation).

This technique is more sensitive than a similar method using trinitrobenzene sulphonate (Palmer \& Peters, 1965), but the decarboxylation method of Lacy \& Crofford (1964) (see also Foley \& Reynolds, 1968) probably remains the most precise automatic method for determining total amino acid concentrations.

\section{REFERENCES}

Foley, T. H. \& Reynolds, M. L. (1968). Technicon 6th Colloquium in Amino Acid Analysis. (In the Press.) Lacy, W. W. \& Crofford, O. B. (1964). J. Lab. clin. Med. 64, 828.

Palmer, D. W. \& Peters, T. Jr (1965). Automation in Analytical Chemistry Technicon Symposia p. 325. Prenton, M. A. \& London, D. R (1967). Technicon 5 th Colloquium in Amino Acid Analysis p. 70.

\section{Identification and estimation of ninhydrin-positive substances in physio- logical fluids}

\section{By G. Leaf, Department of Biochemistry, The University, Glasgow}

Paper for publication not received. 